Europhys. Lett., 63 (3), pp. 419-425 (2003)

\title{
Spin-polarized vacuum tunneling at small gap widths
}

\author{
H. F. Ding $\left({ }^{*}\right)$, W. Wulfhekel $(* *)$, U. Schlickum and J. Kirschner \\ Max-Planck Institut für Mikrostrukturphysik - Weinberg 2, 06120 Halle, Germany
}

(received 17 September 2002; accepted in final form 5 June 2003)

PACS. 72.25. Mk - Spin transport through interfaces.

PACS. 68.37.Ef - Scanning tunneling microscopy (including chemistry induced with STM).

\begin{abstract}
We studied spin-polarized tunneling through a vacuum barrier using spinpolarized scanning tunneling microscopy on $\mathrm{Co}(0001)$. By varying the tip-to-sample distance in a controlled way, the tunneling magnetoresistance, i.e., the tunneling current asymmetry for parallel and antiparallel configuration of tip and sample magnetization, was measured as a function of the gap width. At large gap widths the asymmetry is constant. At gap widths below $\approx 4.5 \AA$, a drop of the asymmetry was found in contrast to predictions of the Jullière model. The drop of the current asymmetry in spin-polarized tunneling is found to correlate with drop of the imaginary electron momentum inside the barrier and we compare our experimental results with the predictions of the Slonczewski model.
\end{abstract}

In 1971, Tedrow and Meservey opened up the field of spin-polarized tunneling (SPT) by studying electron tunneling between a ferromagnet and a superconductor under an applied magnetic field [1]. Later, Jullière found that due to spin-polarized tunneling between two ferromagnetic layers, the tunneling resistance depends on the relative orientation of magnetization of the layers [2]. Since then, SPT has been studied intensively from the fundamental point of view to illuminate the underlying physical mechanisms [3-9] and has stimulated many new applications [10-13]. Nevertheless, SPT is still far from complete understanding. The difficulties to obtain a coherent picture of the effect are partly related to the complex structure of the tunneling junctions, often containing poorly characterized amorphous barriers causing higher-order effects in SPT [13-15].

In this letter, we study SPT across the tunable vacuum barrier of a spin-polarized scanning tunneling microscope (Sp-STM) [16-18]. Sp-STM provides an ideal model system to study SPT as it has a simple vacuum barrier, whose width can be controlled in a precise way, and allows as well to use atomically clean surfaces as electrodes. In contrast to a variety of different models for SPT, there is consensus that the tunneling magnetoresitance (TMR) effect is a consequence of the exchange splitting of the band structure of the ferromagnetic electrodes which leads to an unbalanced distribution of majority and minority electrons at the Fermi energy. Assuming conservation of the electron spin during tunneling, this imbalance leads to different tunneling currents $I$ for parallel (p) and antiparallel (ap) magnetic configuration.

(*) Present address: Argonne National Laboratory - 9700 S. Cass Avenue, Argonne, IL 60439, USA.

$(* *)$ E-mail: wulf@mpi-halle.mpg.de

(C) EDP Sciences 
Jullière [2] proposed a phenomenological model that relates the asymmetry $\delta$ of the tunneling current to the spin polarization $P_{f}(f=1,2)$ of the two ferromagnetic electrodes:

$$
\delta=\frac{I_{\mathrm{p}}-I_{\mathrm{ap}}}{I_{\mathrm{p}}+I_{\mathrm{ap}}}=P_{1} P_{2} .
$$

This model has been commonly used in many studies [3,4], sometimes with the extension that $P_{f}$ is the polarization of the ferromagnet/barrier interface [6-9]. Later, Slonczewski calculated the TMR in the approximation of Bloch waves and showed that $\delta$ does not only depend on the polarizations $P_{f}$ of both electrodes/interfaces, but also on the momenta of the electrons in the electrodes and the barrier [19]. MacLaren et al. investigated the validity of both models by comparing them with first-principle calculations of $\delta$ between iron electrodes separated by a vacuum barrier showing that Slonczewski's model gives a better description [20]. We here focus on measurements of the current asymmetry $\delta$ as a function of the vacuum barrier width. We present evidence for a correlation between $\delta$ and the imaginary electron momentum inside the barrier which is in qualitative agreement with the model given by Slonczewski.

A bulk single crystalline $\mathrm{Co}(0001)$ sample is selected for the measurements. All experiments were performed in ultra-high vacuum $\left(p=5 \times 10^{-11} \mathrm{mbar}\right)$. The vacuum chamber was equipped with an Auger electron spectrometer (AES), low-energy electron diffraction (LEED) and a Sp-STM [16-18]. Both the Co(0001) sample and the magnetic tip were cleaned in situ by sputtering with $1 \mathrm{keV} \mathrm{Ar}^{+}$ions. The sample was annealed afterwards to $570 \mathrm{~K}$. In AES spectra no traces of contaminations could be found. LEED images showed the expected sixfold diffraction pattern with sharp spots and low background intensity. The detailed principle and technique of Sp-STM have been addressed in previous publications [16-18]. During scanning, an alternating current of $\approx 10 \mathrm{~mA}$ at $40 \mathrm{kHz}$ was passed through a small coil wound around the magnetic tip to periodically switch the longitudinal magnetization of the tip. This results in variations of the tunneling current due to the TMR effect [2]. These variations were detected with a phase-sensitive lock-in amplifier to map the sample magnetic structure. In contrast to the method of Bode et al. [21], not the differential conductance but the change of the tunneling current, i.e., the asymmetry $\delta$, is measured. Tunneling images of both the topography and the magnetic structure were recorded simultaneously at room temperature. Figure 1a shows the typical dendritic-like perpendicular domain pattern of $\mathrm{Co}(0001)$ that was observed [22], similar to that seen with scanning electron microscopy with polarization analysis [23] and magnetic-force microscopy (MFM) [24]. The magnetic origin was crosschecked by applying an external magnetic field. The domain pattern can be displaced while the topography image does not shift. The exposure of a few Langmuir of oxygen causes the magnetic signal to disappear as expected for spin-polarized tunneling. Further, magnetic contrast due to mechanisms similar to MFM has been excluded [24] and distance changes of the tip vs. the sample due to magnetostriction are experimentally and theoretically shown to be below $0.1 \mathrm{pm}$ ruling out a magnetic contrast due to magnetostriction [25]. Finally, the deposition of a few monolayers of $\mathrm{Au}$ on $\mathrm{Co}(0001)$ causes a fading of the contrast, i.e., the TMR, as observed for spin-polarized tunneling in Au-seeded planar tunneling junctions [26]. To study $\delta$ as a function of the barrier width, we zoomed into small areas which contain only two domains separated by narrow domain walls (see fig. 1b). This domain wall is found to correspond to the domain wall across two canted surface domains (see fig. 1c and, for more detail, ref. [22]). As the TMR effect is a linear function of the scalar product between the magnetization of the tip and the sample $[19,27]$, any variation of the TMR leads to the same variations in the contrast due to SPT observed between the two domains. To study the dependence of the TMR on the gap width, we took line scans across a domain wall as a function of the gap resistance by changing the feedback current. A lower current, i.e., higher tunneling resistance, corresponds to a wider 

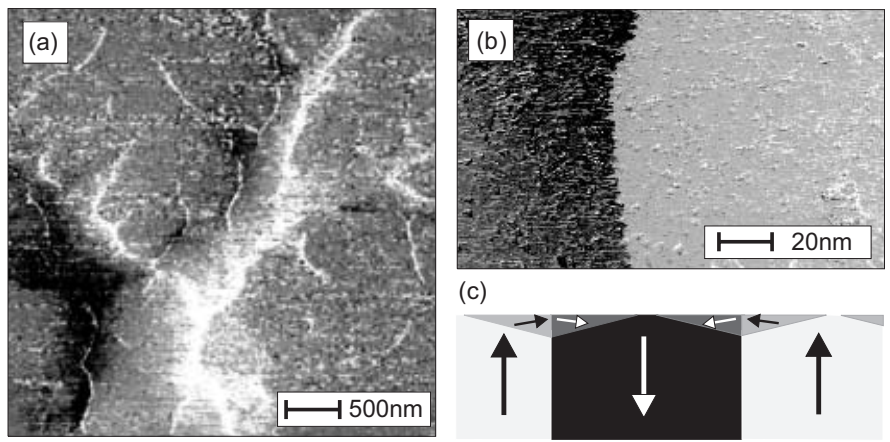

(c)

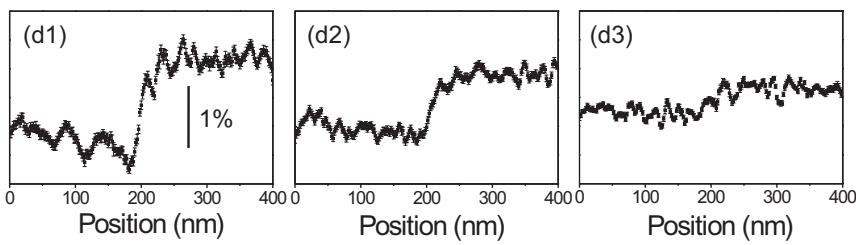

Fig. 1 - (a) Magnetic domain image on Co(0001) obtained by Sp-STM. (b) A sharp domain wall at the end of a branch in high magnification. (c) Schematic cross-section of the closure domain pattern of Co. And line scans across the domain wall with different tunneling resistances: (d1) $2 \times 10^{7} \Omega$, (d2) $4 \times 10^{6} \Omega$, (d3) $1 \times 10^{6} \Omega$. The bias voltage is $20 \mathrm{mV}$ in all images.

gap. In that way, the modulated current caused by SPT was measured as a function of the tunneling current and the asymmetry $\delta$ is calculated from their ratio. To reduce the noise, we imaged the same line repeatedly (200 times) and averaged the data. Figure $1 \mathrm{~d}(1-3)$ presents 3 line profiles at different tunneling resistances. From the profiles, it is obvious that $\delta$ is a function of the tip-to-sample distance.

Figure 2 shows the dependence of $\delta$ as a function of the tunneling resistance measured over a wider range. Above $10 \mathrm{M} \Omega$, the TMR is constant and drops below this resistance continuously. Assuming a contact resistance of $\approx 24 \mathrm{k} \Omega$, the resistance of $10 \mathrm{M} \Omega$ corresponds to a tip-to-sample distance of $\approx 4.5 \AA[28,29]$.

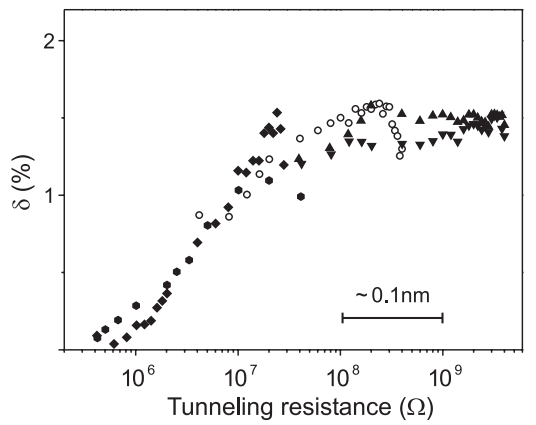

Fig. 2 - Drop of the TMR $\delta$ as a function of tunneling resistance. The data has been obtained with a set of different tips and bias voltages. A reduction of the tunneling resistance by one order of magnitude corresponds roughly to an approach of the tip by $\approx 0.1 \mathrm{~nm}$. 
In the model given by Jullière, $\delta$ does not depend on the barrier width, i.e., the tip-tosample distance in our experiments. It only depends on the spin polarization of the ferromagnetic electrodes $P_{f}$. Obviously, this model cannot explain our experimental finding as it would suggest that either the tip or the sample magnetization drops during the approach. A more realistic model was proposed by Slonczewski, who calculated the TMR in the approximation of Bloch waves for free electrons. He pointed out that $\delta$ does not only depend on the spin polarization of the two electrodes but also on the electron momentum inside the barrier. As a consequence, he proposed to use an effective spin polarization of the ferromagnetic electrode and barrier couple $P_{f b}$ instead of the spin polarization of the ferromagnetic electrode $P_{f}$ to describe the current asymmetry,

$$
\delta=P_{1 b} P_{2 b}
$$

where $P_{1 b}$ and $P_{2 b}$ are defined as

$$
P_{1(2) b}=\frac{\left(k_{1(2)}^{\uparrow}-k_{1(2)}^{\downarrow}\right)}{\left(k_{1(2)}^{\uparrow}+k_{1(2)}^{\downarrow}\right)} \frac{\left(\kappa^{2}-k_{1(2)}^{\uparrow} k_{1(2)}^{\downarrow}\right)}{\left(\kappa^{2}+k_{1(2)}^{\uparrow} k_{1(2)}^{\downarrow}\right)},
$$

in which $i \kappa$ is the imaginary wave vector in the barrier and $k_{f}^{\uparrow}$ and $k_{f}^{\downarrow}$ are the wave vectors inside the ferromagnetic electrodes for majority and minority charges. In the case that $\kappa$ changes with the tip-to-sample distance, $\delta$ also changes. Especially, if $\kappa^{2}=k_{f}^{\uparrow} k_{f}^{\downarrow}$, the current asymmetry is zero irrespective of the spin polarization of the electrodes, in contrast to Jullière's model. At small bias voltages, $\kappa$ can be obtained from the tunneling current $I$ as a function of the tip-to-sample distance according to the following equation:

$$
\kappa\left(\AA^{-1}\right)=-\frac{1}{2} \frac{\mathrm{d} \ln I}{\mathrm{~d} S},
$$

where $S$ (in $\AA$ ) is the barrier width. In a combined experiment, we simultaneously determined $\kappa$ and $\delta$ as a function of gap width. In each pixel of scan line across the wall, the feedback loop was opened for a short time and the tip was approached/retracted continuously up to a displacement of $0.1 \mathrm{~nm}$ from its original position while measuring the tunneling current and the modulated current caused by SPT. Figure 3a shows the tunneling currents vs. the tip displacement obtained on the two domains on both sides of the domain wall at $20 \mathrm{mV}$ sample bias. Both of the tunneling currents identically increase nearly exponentially when the tip approaches the sample surface as expected. Figure $3 \mathrm{~b}$ presents $\delta$ as a function of the tip displacement. It shows the drop of $\delta$ at gap distances corresponding to a tunneling resistance smaller than $10 \mathrm{M} \Omega(20 \mathrm{mV}, 2 \mathrm{nA})$. As we used a constant bias voltage [30], the variation of $\delta$ has to be attributed to the change of the tip-to-sample distance. Figure 3c shows $\kappa$ $v s$. the tip displacement calculated from the data shown in fig. 3a. It is nearly constant at large tip-sample separations and decreases when the tip further approaches the sample. This is in agreement with a drop of the effective work function, when approaching STM tips to the surface $[28,29]$, before an Ohmic contact is formed. The observed change of $\kappa$ behaves similarly to the tip-to-sample distance-dependent asymmetry $\delta$ shown in fig. 3b. This suggests a correlation between these two.

To quantify the influence of $\kappa$ on the current asymmetry $\delta$, we performed calculations in the model proposed by Slonczewski [19]. With $\kappa$ measured above, the current asymmetry $\delta$ as a function of the tip displacement can be calculated by applying the formula given in eqs. (2) and (3). The wave vectors for spin-up and spin-down electrons for Co in the freeelectron approximation can be calculated from the exchange energy ( $1 \mathrm{eV}$ from [31]) and the 


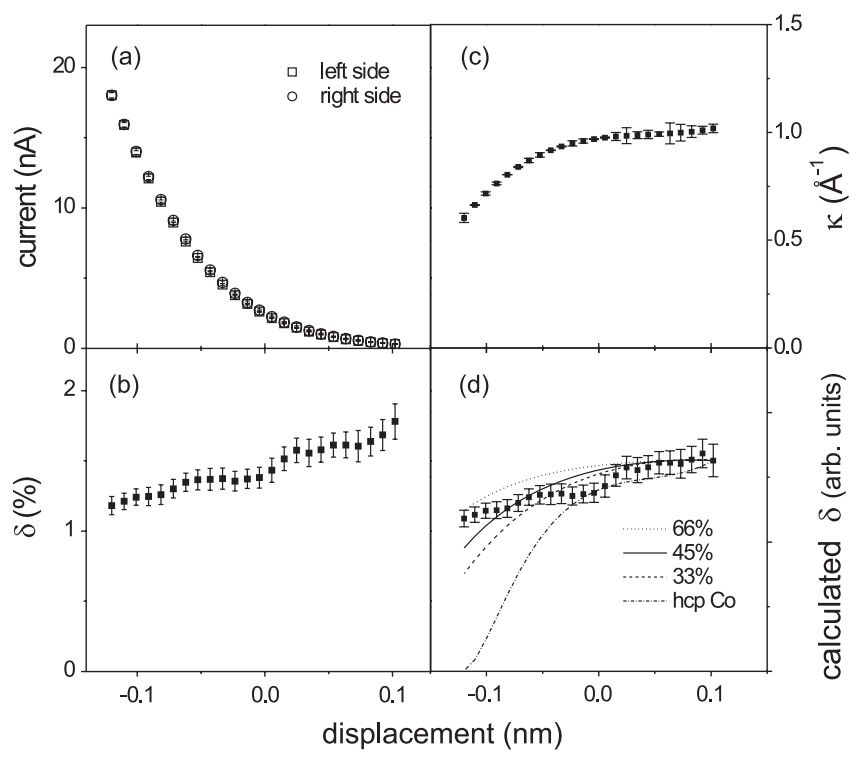

Fig. 3 - (a) Tunneling currents as a function of vertical tip displacement obtained at the left and the right side of the domain wall. Negative displacements indicate an approach of the tip from the original position corresponding to tunneling parameters of $2 \mathrm{nA}$ at $20 \mathrm{mV}$. (b) Simultaneously measured current asymmetry $\delta$. (c) The tip-to-sample distance-dependent wave vector $\kappa$ calculated from the tunneling current. (d) Comparison between the measured asymmetry $\delta$ and those calculated using the free-electron model for 3 different values of the spin polarization and the electron momenta for hcp $\mathrm{Co}(0001)$ with the wave vector $\kappa$. The calculated curves are normalized to the measured $\delta$ at large tip-to-sample distance.

spin polarization [32]. Since there is no direct measurement for the spin polarization of single crystal $\mathrm{Co}(0001)$, we calculate $\delta$ for 3 different values. The 3 values are, $33 \%$ chosen from an early measurement by Meservey and Tedrow [33], 45\% chosen from recently reported values by Moodera et al. [34], and $66 \%$ selected just for comparison reasons. The same values are chosen for the magnetic tip as the tip material is dominated by Co $(\approx 92 \%)$. The values mentioned above were obtained with $\mathrm{Al}_{2} \mathrm{O}_{3}$ barriers with a barrier height of $\approx 2.5 \mathrm{eV}[34]$. With these 3 different sets of wave vectors and the distance-dependent $\kappa$, the asymmetry as a function of the tip-to-sample distance are calculated. Figure 3d presents the results of these calculations for free electrons. For comparison, the experimentally measured tip-tosample distance-dependent asymmetry (filled squares) are shown in this figure as well. All curves are normalized to the value of $\delta$ at large tip-to-sample distance. The figure shows that the calculations for all three spin polarization values reproduce qualitatively the drop of the asymmetry with the tip approaching, even though the polarizations are varying by a factor of 2. At small tip-sample separations, the calculated asymmetry deviates from the measured one. This deviation may be due to two facts. First, the free-electron approximation neglects the details of the band structure of Co, i.e., $k^{\uparrow}$ and $k^{\downarrow}$ deviate from that obtained in the free-electron approximation. But due to the fact that tunneling is restricted to the electrons near the Fermi level, the detailed shape of the bands well below or above the Fermi level has little influence on $\delta$. A steeper drop of $\delta$ with the distance is obtained at small distances (see fig. 3d), when $k^{\uparrow}$ and $k^{\downarrow}$ at the Fermi energy are taken from the observed band structure of Co [35], i.e. Co is not treated in the free-electron approximation but the experimental band 
structure is considered. Secondly and most importantly, Slonszewski treated the tunneling in a quasi-one-dimensional limit for electrons with no transverse momentum. When $\delta$ is estimated with a fully three-dimensional treatment, the drop of the TMR is reduced. This effect is more dominant at small gap width when tunneling proceeds also via states with nonvanishing transverse momenta [19]. Especially, the condition $\kappa^{2}=k_{f}^{\uparrow} k_{f}^{\downarrow}$ cannot be fulfilled for all momenta in 3 dimensions and the TMR is not expected to vanish completely.

Besides the reduction of the asymmetry due to a reduced $\kappa$, two other mechanisms might also contribute to the distance dependence. When the tip is approached to the sample surface, the tip and sample interact with each other, influencing their spin polarizations. The interaction between an $\mathrm{Fe}(001)$ surface and an Fe tip has been investigated by first-principle theory [36], showing a decrease in the spin polarization when the tip-to-sample separation is below $2.88 \AA$. A similar mechanism could be operative in our case with a stronger suppression of the spin polarization at much larger distances. Second, the Slonszewski model within its free-electron approximation does not distinguish between $s$-, $p$ - or $d$-electrons, while the real density of states in front of the surface is a mixture of all three. The balance between $s^{-}, p-$, and $d$-electron tunneling may, however, change with the gap width and by this influence the effective spin polarization and the TMR [37,38]. Full theoretical calculations are needed to study the importance of this mechanism.

The reduction of the TMR with decrease of the gap width is also found for bias voltages other than $20 \mathrm{mV}$ and shows a weak voltage dependence. The drop is most pronounced at $200 \mathrm{mV}$ [39]. The voltage dependence is likely due to surface states of $\mathrm{Co}(0001)$ that contribute to the tunneling process, changing the electron momenta of the relevant states of the electrodes.

In conclusion, using a model system with a tunable vacuum barrier we simultaneously measured both the tip-to-sample distance-dependent current asymmetry $\delta$ and the imaginary wave vector inside the barrier $\kappa$. We observe a correlated drop of both $\kappa$ and $\delta$ at small distances and suggest that the TMR is dependent on the momentum inside the barrier. The observed behavior is compared to the predictions of Slonczewski in the framework of free electrons and reasonably good agreement is found. Our experimental findings also give a guidance for the practical measurement of Sp-STM and fabrication of high-density magnetic random access memory (M-RAM). For optimal performance of Sp-STM, it is necessary to work at large tip-to-sample distance $(R>10 \mathrm{M} \Omega)$ to achieve a high magnetic contrast. For the fabrication of high-density M-RAM using tunneling junctions, small resistance-area products are desired $[13,40]$. Our measurements suggest that, with a reduction of the barrier thickness, the resistance cannot be scaled down arbitrary. For extremely thin oxide barriers, a similar drop of $\delta$ is expected reducing the signal-to-noise ratio of a M-RAM bit.

\section{REFERENCES}

[1] Tedrow P. M. and Meservey R., Phys. Rev. Lett., 26 (1971) 192.

[2] Jullière M., Phys. Lett. A, 54 (1975) 225.

[3] Moodera J. S., Kinder L. R., Wong T. M. and Meservey R., Phys. Rev. Lett., 74 (1995) 3273.

[4] Lu Y., Altman R. A., Marley A., Rishton S. A., Troullloud P. L., Xiao G., Gallagher W. J. and Parkin S. S. P., Appl. Phys. Lett., 70 (1997) 2610.

[5] Zhang S., Levy P. M., Marley A. C. and Parkin S. S. P., Phys. Rev. Lett., 79 (1997) 3744.

[6] Teresa J. M. D., Barthélémy A., Fert A., Contour J., Montaigne F. and Seneor P., Science, 286 (1999) 507.

[7] Sharma M., Wang S. X. and Nickel J. H., Phys. Rev. Lett., 82 (1999) 616. 
[8] LeClair P., Kohlhepp J. T., Swagten H. J. M. and De Jonge W. J. M., Phys. Rev. Lett., 86 (2001) 1066.

[9] Yuasa S., Sato T., Tamura E., Suzuki Y., Yamamori H., Ando K. and Katayama T., Europhys. Lett., $\mathbf{5 2}$ (2000) 344.

[10] Tondra M., Daughton J. M., Nordman C., Wang D. and Taylor J., J. Appl. Phys., 87 (2000) 4679.

[11] Scheuerlein R., Gallagher W., Parkin S. S. P., Lee A., Ray S., Robertazzi R. and REOHR W., IEEE International Solid-State Circuits Conference, Digest of Technical Papers, 2000.

[12] Freitas P. P., Cardoso S., Sousa R., Wanjun K., Ferreira R., Chu V. and Conde J. P., IEEE Trans. Magn., 36 (2000) 2796.

[13] Boeve H., Girgis E., Schelten J., Boeck J. D. and Borghs G., Appl. Phys. Lett., 76 (2000) 1048.

[14] Zhang J. and White R., J. Appl. Phys., 83 (1998) 6512.

[15] Tsymbal E. Y. and Pettifor D., J. Appl. Phys., 85 (1999) 5801.

[16] Wulfhekel W. and Kirschner J., Appl. Phys. Lett., 75 (1999) 1944.

[17] Wulfhekel W., Ding H. F. and Kirschner J., J. Appl. Phys., 87 (2000) 6475.

[18] Wulfhekel W., Ding H. F., Lutzke W., Steierl G., Vàzquez M., Marìn P., Hernando A. and Kirschner J., Appl. Phys. A, 72 (2001) 463.

[19] Slonczewski J. C., Phys. Rev. B, 39 (1989) 6995.

[20] Maclaren J. M., Zhang X. G. and Butler W. H., Phys. Rev. B, 56 (1997) 11827.

[21] Bode M., Getzlaff M. and Wiesendanger R., Phys. Rev. Lett., 81 (1998) 4256.

[22] Ding H. F., Wulfhekel W. and Kirschner J., Europhys. Lett., 57 (2002) 100.

[23] Unguris J., Scheinfein M. R., Celotta R. C. and Pierce D., Appl. Phys. Lett., 55 (1989) 2553.

[24] Ding H. F., Wulfhekel W., Chen C., Barthel J. and Kirschner J., Mater. Sci. Eng. B, 84 (2001) 96.

[25] Wulfhekel W., Hertel R., Ding H. F., Steierl G. and Kirschner J., J. Magn. 83 Magn. Mater., 249 (2002) 368.

[26] Moodera J. S., Taylor M. E. and Meservey R., Phys. Rev. B, 40 (1989) 11980.

[27] Miyazaki T. and Tezuka N., J. Magn. \& Magn. Mater., 139 (1995) L231.

[28] Gimzewski J. K. and Möller R., Phys. Rev. B, 36 (1987) 1284.

[29] Kuk Y. and Silverman P. J., J. Vac. Sci. Technol. A, 8 (1990) 289.

[30] Due to the low resistance of $4 \mathrm{~K} \Omega$ of the $I-V$ converter of the STM, it is ensured that even for relatively small tunnel resistances, the tunneling bias is kept constant.

[31] Stearns M., J. Magn. ES Magn. Mater., 5 (1977) 167.

[32] Moodera J. S., Nowak J., Kinder L. R., Tedrow P. M., van de Veerdonk R. J. M., Smits B. A., van Kampen M., Swagten H. J. M. and De Jonge W. J. M., Phys. Rev. Lett., 83 (1999) 3029.

[33] Meservey R. and Tedrow P. M., Solid State Commun., 11 (1972) 333.

[34] Moodera J. S. and Mathon G., J. Magn. 63 Magn. Mater., 200 (1999) 248.

[35] Wetli E., Kreutz T. J., Schmid H., Greber T., Osterwalder J. and Hochstrasser M., Surf. Sci., 402-404 (1998) 551.

[36] Fang C. M., de Groot R. A., Bischop M. M. J. and van Kempen H., Surf. Sci., 436 (1999) L648.

[37] Alvarado S. F., Phys. Rev. Lett., 75 (1995) 513.

[38] Tsymbal E. Y. and Pettifor D. G., J. Phys. Condens. Matter, 9 (1997) L411.

[39] Ding H. F., Wulfhekel W., Henk J., Bruno P. and Kirschner J., Phys. Rev. Lett., 90 (2003) 116603.

[40] Convington M., Nowak J. and Song D., Appl. Phys. Lett., 76 (2000) 3965. 